\title{
Hemodilution, Tonicity, and Blood Viscosity *
}

\author{
Peter W. Rand and Eleanor Lacombe \\ (From the Cardiovascular Research Laboratory, Maine Medical Center, Portland, Mainc)
}

Since the introduction of intravenous therapy, fluids containing glucose or saline in relatively isotonic concentrations have far exceeded others in use. Within the last decade new techniques in many areas of diagnosis and treatment have required that fluids of various tonicity and viscosity be introduced into the blood stream with such rapidity or in such great volume as to preclude adequate dilution with the circulating cells and plasma.

Several in vivo studies (1-4) have demonstrated the effect of hypertonic solutions on blood flow. Some authors (5-7) have noted circulatory changes, especially in the pulmonary vascular bed, secondary to the injection of angiographic contrast media, which are both hypertonic and highly viscous. Others (8-11) have commented on the benefits of gross dilution of perfused blood during extracorporeal support for open-heart surgery, and some data have been presented concerning the flow properties of blood diluted with solutions of dextrose (12) or dextran $(13,14)$.

At any given shear rate or temperature it is the concentration of red cells which primarily affects the viscosity of whole blood. Viscosity rises with an increasing hematocrit. While investigating the effects of various intravenous solutions on the hematocrit and viscosity of whole blood in vitro, we have noted that under certain conditions a drop in hematocrit has been accompanied by a rise in viscosity, whereas under other circumstances an increase in hematocrit has been accompanied by a fall in viscosity. The significance of these findings in respect to the flow of blood exposed to diluents of varying osmolality and viscosity appears to be of sufficient importance to warrant reporting at this time.

\footnotetext{
* Submitted for publication May 1, 1964; accepted July 21, 1964.

Supported by grant HE 07984 from the National Institutes of Health and by the Maine Heart Association.
}

\section{Methods}

The principal experiments were designed to measure changes in the viscosity of blood after its in vitro dilution by solutes that alter either plasma tonicity or plasma viscosity. To study the effects of tonicity, compounds were selected that vary in their ability to permeate the red cell membrane and hence in their ability to produce cell distention or crenation by osmotic gradients; all are components of common intravenous fluids. Wide ranges of dilution and concentration were explored. The parameters measured, in addition to blood and plasma viscosity, included osmolality, water content, and hematocrit. Since the initial number of red cells remained constant in each case, variations in hematocrit from the level predicted on the basis of equivalent isotonic dilution indicated changes in cell size. Similar methods were used to study the response of blood viscosity to changes in plasma viscosity, except that isotonic solutions of high and low molecular weight dextrans were the diluents.

Specifically, to compare hypotonic and isotonic dilutions, deionized water or isotonic saline $(0.9 \mathrm{mg}$ per 100 $\mathrm{ml}, 287$ mOsm per $\mathrm{kg}$ ) was added to samples of fresh human blood with hematocrit adjusted to 45 . The following proportions were used: $1 \mathrm{ml}$ diluent to $19 \mathrm{ml}$ blood $(1: 20), 2 \mathrm{ml}$ diluent to $18 \mathrm{ml}$ blood $(1: 10)$, and $4 \mathrm{ml}$ diluent to $16 \mathrm{ml}$ blood (1:5). Measurements were made on the original (control) and diluted samples after 30 minutes. These experiments were performed in quadruplicate on blood from different normal donors.

To evaluate the effect of cell distention or crenation on blood viscosity, $1: 20,1: 10$, and $1: 5$ dilutions were made as described above with multiple concentrations of saline $(0.5,0.85,3.0,5.0 \%)$, mannitol $(5.0,12.5$, $25 \%)$, dextrose $(5.0,10.0,20.0,50.0 \%)$, and urea $(0.85,1.7,4.2,10.0 \%)$. The control sample for each dilution of each compound tested was blood to which an equivalent amount of plasma had been added. The hematocrit of donor blood was adjusted before the addition of plasma so that each control sample would have a hematocrit of 40 (42.1, 44.4, and 50 for $1: 20$, $1: 10$, and $1: 5$ dilutions, respectively). This portion of the study included 54 separate dilutions representing triplicate determinations for each solute in the hypotonic and isotonic ranges and at least 6 determinations for each in the hypertonic concentrations. For each compound the responses to increasing concentrations were the same at all dilutions, but the magnitude of response varied in proportion to the degree of dilution. To avoid duplication, we have selected data from the 1:10 dilutions for detailed analysis. 
TABLE I

Osmolality and viscosity of test solutions

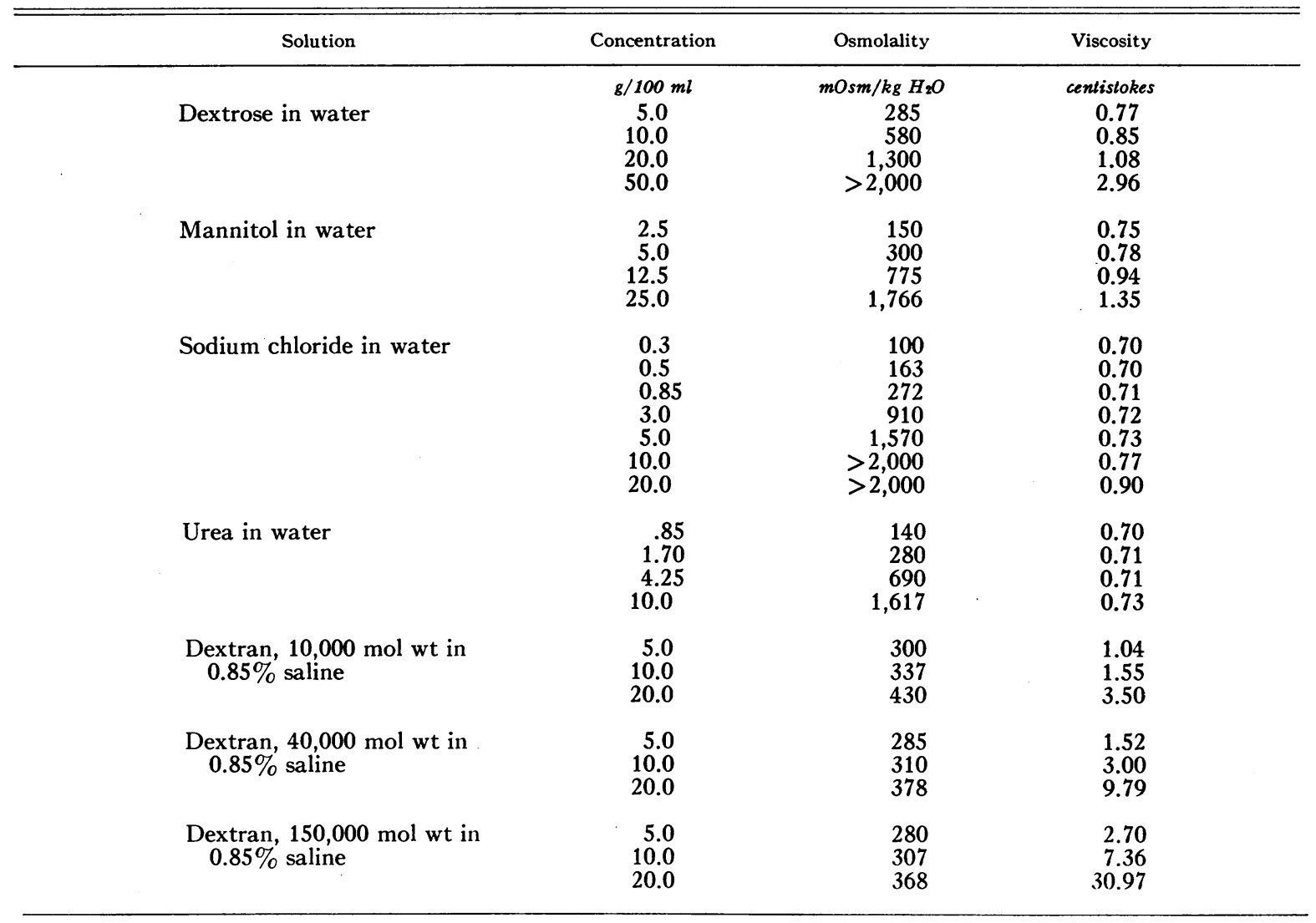

The same procedure was employed to investigate the effects of the dextrans, except that the diluents consisted of 5,10 , and $20 \%$ solutions of dextrans of 10,000 , 40,000 , and $150,000 \mathrm{~mol}$ wt made up in isotonic saline. Osmolality and hematocrit thus remained essentially constant throughout each study. Dilutions of $1: 20$, $1: 10$, and $1: 5$ were studied, providing triplicate determinations at each concentration. For each dextran the response varied in magnitude only, in proportion to the degree of dilution. Data from the 1:10 dilutions are reported.

The osmolality and viscosity of each concentration of diluent appear in Table I. Note the wide range of osmolality in the electrolyte and crystalloid solutions and the high viscosities of the dextran solutions. For each experiment, fresh venous blood was drawn with $50-\mathrm{ml}$ siliconized syringes containing $0.37 \mathrm{ml}$ heparin, $1: 1,000$. To avoid variations caused by duration of the red cells' exposure to the test solutions, studies were initiated on each sample 30 minutes after initial mixing.

Samples were measured for whole blood viscosity and hematocrit, for plasma viscosity, osmolality, water content, and specific gravity. Whole blood viscosity (centi- poise) ${ }^{1}$ was measured at shear rates from 212 seconds $^{-1}$ to 5 seconds $^{-1}$ at $37.0^{\circ} \mathrm{C}$ with a Brookfield micro coneplate viscometer after the method of Rand, Lacombe, Hunt, and Austin (15). Although complete information regarding the response of viscosity at all shear rates has been presented in some instances, values at 106 seconds $^{-1}$ have been used to demonstrate viscosity changes between samples. This particular shear rate was selected because it is slow enough to reflect the shear-rate-dependent character of whole blood yet rapid enough to provide

1 Viscosity, by definition, is shear stress/shear rate (dyne-sec- $\mathrm{cm}^{-2}$, or poise). $1 / 100$ poise $=1$ centipoise (cp). Since blood viscosity varies with shear rate, it must be measured with a variable-speed rotational viscometer such as used here. These instruments measure shear stress, from which viscosity, in centipoise, is calculated. Plasma viscosity is most practically measured in a capillary tube viscometer. Viscosity is calculated from the rate of fall of a fixed volume of test liquid, which is related to density, other factors remaining constant. The units are centistokes (cs). 1 centistoke $=1$ centipoise/density. 
excellent reproducibility throughout the hematocrit range involved. Normal values at this shear rate $(\mathrm{Ta}-$ ble II) and normal viscosity-shear-rate curves for each hematocrit were derived from data previously obtained in this laboratory (15).

Plasma viscosity (centistokes) was measured at $37^{\circ} \mathrm{C}$

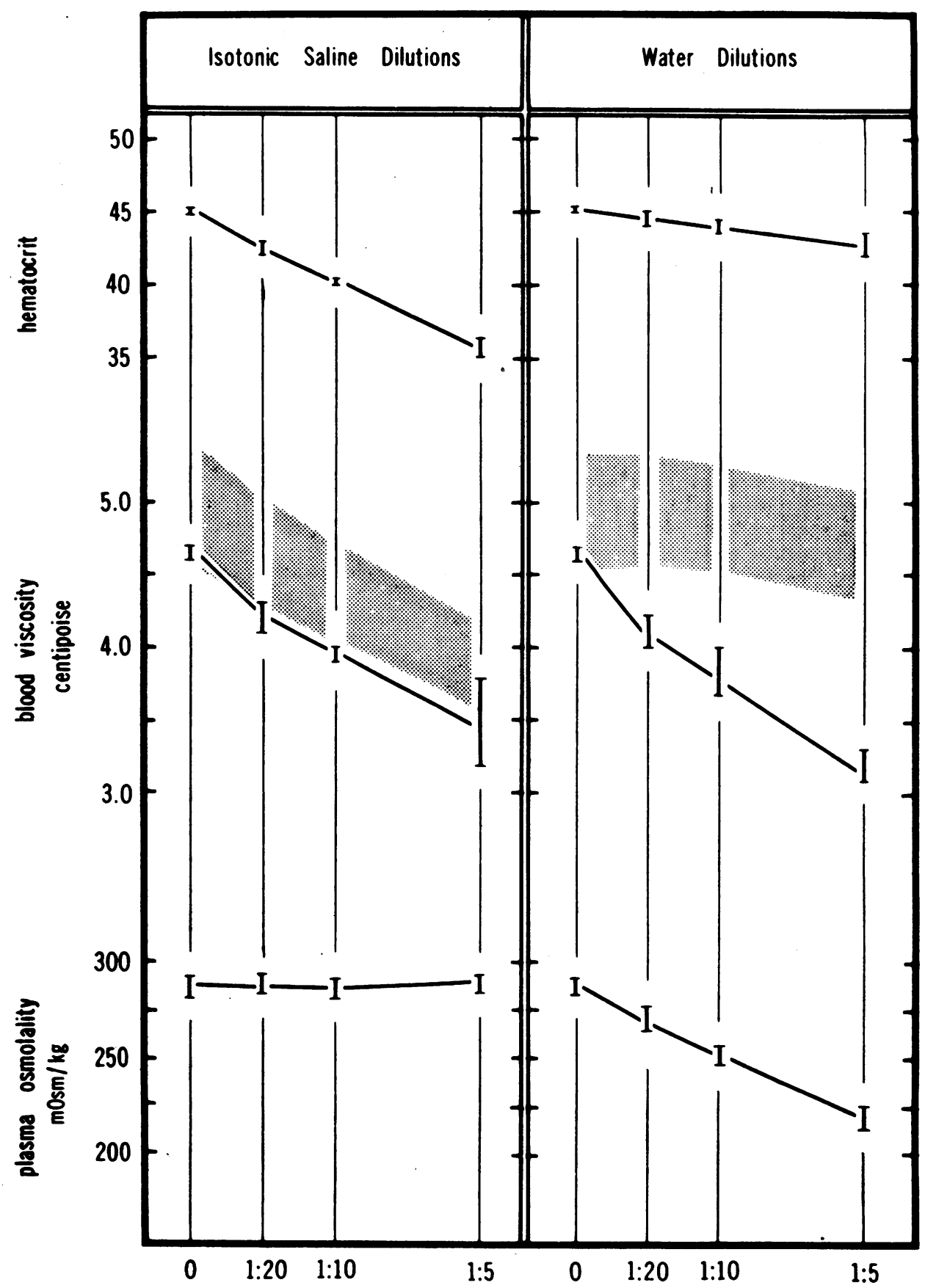

Fig. 1. A COMPARISON OF THE EFFECTS OF ISOTONIC AND HYPOTONIC DILUTIONS ON HEMATOCRIT AND BLOOD vISCosity. The shaded areas represent \pm 1 SD of the normal blood viscosity at the hematocrits produced. Vertical lines indicate the range of values from all experiments. Although hematocrits remain elevated with increasing hypotonic dilution due to increased red cell size, blood viscosity falls to the same extent as in equally diluted isotonic samples. 


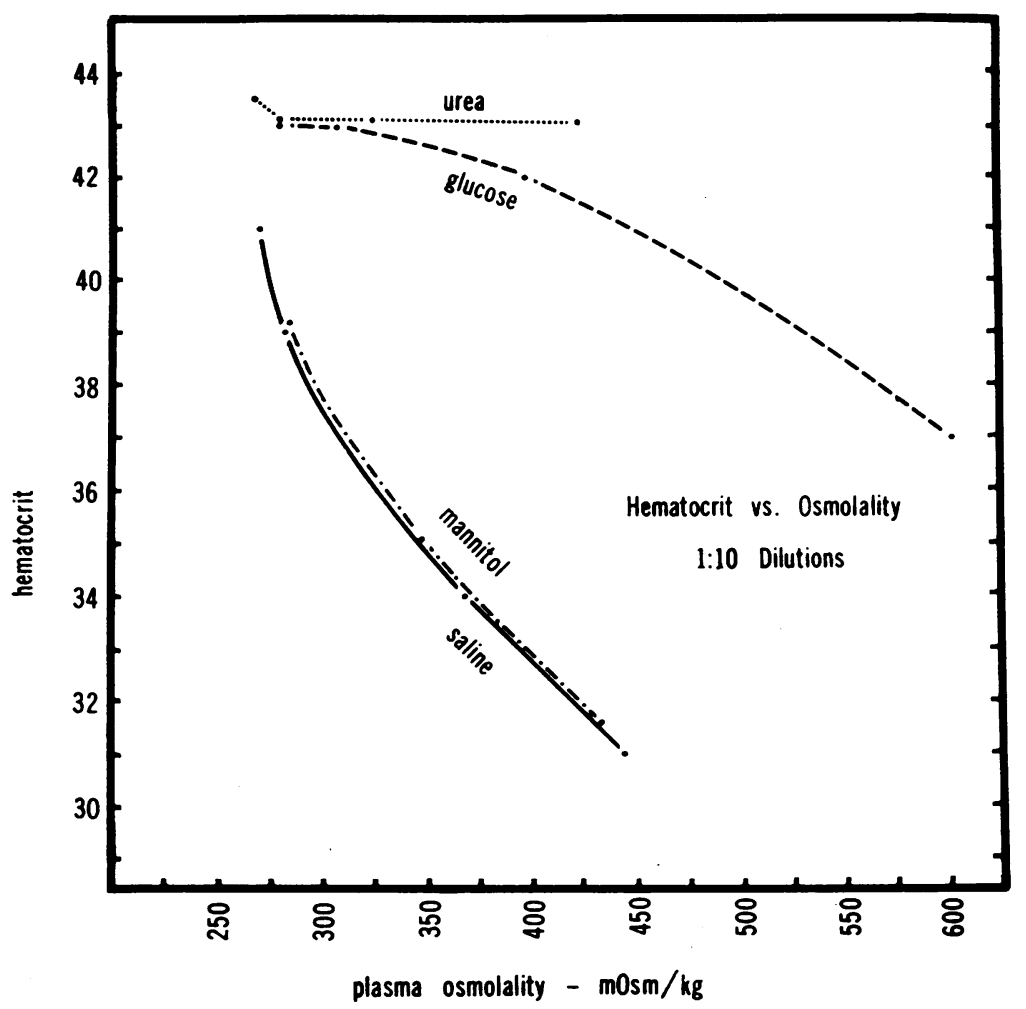

Fig. 2. The RESPONSE OF RED CELL SIZE TO INCREASING CONCENTRATIONS of SOLUTES TESTED. Since red cell concentration and degree of dilution are constant for each compound, any change in hematocrit reflects a change in cell volume.

with Ostwald capillary tube viscometers. Since the viscosity of plasma at this temperature exhibits no shearrate dependence (16), this instrument may be used. Calibration was performed according to the directions of Hardy (17) using deionized water, absolute ethyl alcohol, and standard viscosity oil $\mathrm{H}$ from the National
Bureau of Standards. Viscosity values were reproducible to within extremely fine limits $(2 \mathrm{SD}= \pm$ $0.004 \mathrm{cs}$ ).

Water contents (g water per $100 \mathrm{~g}$ plasma) were determined gravimetrically by the desiccation of $2.00 \mathrm{ml}$ (Van Slyke pipette) of plasma. Osmolality was meas-

TABLE II

Hematocrit-viscosity relationships for normal human blood at $37^{\circ} \mathrm{C}$, shear rate 106 seconds $^{-1}$ (15)

\begin{tabular}{|c|c|c|c|c|c|c|c|c|}
\hline $\begin{array}{c}\text { Hemato- } \\
\text { crit }\end{array}$ & Viscosity & SD & $\underset{\text { crit }}{\text { Hemato- }}$ & Viscosity & SD & $\underset{\text { crit }}{\text { Hemato- }}$ & Viscosity & SD \\
\hline & centipoise & & & centipoise & & & centipoise & \\
\hline 60 & 7.2 & \pm 0.48 & 46 & 5.1 & & 32 & 3.5 & \\
\hline 59 & 7.1 & & 45 & 5.0 & & 31 & 3.4 & \\
\hline 58 & 6.9 & & 44 & 4.9 & & 30 & 3.4 & \\
\hline 57 & 6.7 & & 43 & 4.7 & & 29 & $\begin{array}{l}0.4 \\
3.3\end{array}$ & \\
\hline 56 & 6.6 & & 42 & 4.6 & & 28 & 3.2 & \\
\hline 55 & 6.4 & & 41 & 4.5 & & 27 & 3.1 & \\
\hline 54 & 6.2 & & 40 & 4.4 & \pm .38 & 26 & 3.0 & \\
\hline 53 & 6.1 & & 39 & 4.3 & & 25 & 2.9 & \\
\hline 52 & 5.9 & & 38 & 4.2 & & 24 & 2.9 & \\
\hline 51 & 5.8 & & 37 & 4.0 & & 23 & 2.8 & \\
\hline 50 & 5.7 & & 36 & 3.9 & & 22 & 2.7 & \\
\hline 49 & 5.5 & & 35 & 3.8 & & 21 & 2.6 & \\
\hline 48 & 5.4 & & 34 & 3.7 & & 20 & 2.6 & \pm .16 \\
\hline 47 & 5.3 & & 33 & 3.6 & & & & \\
\hline
\end{tabular}




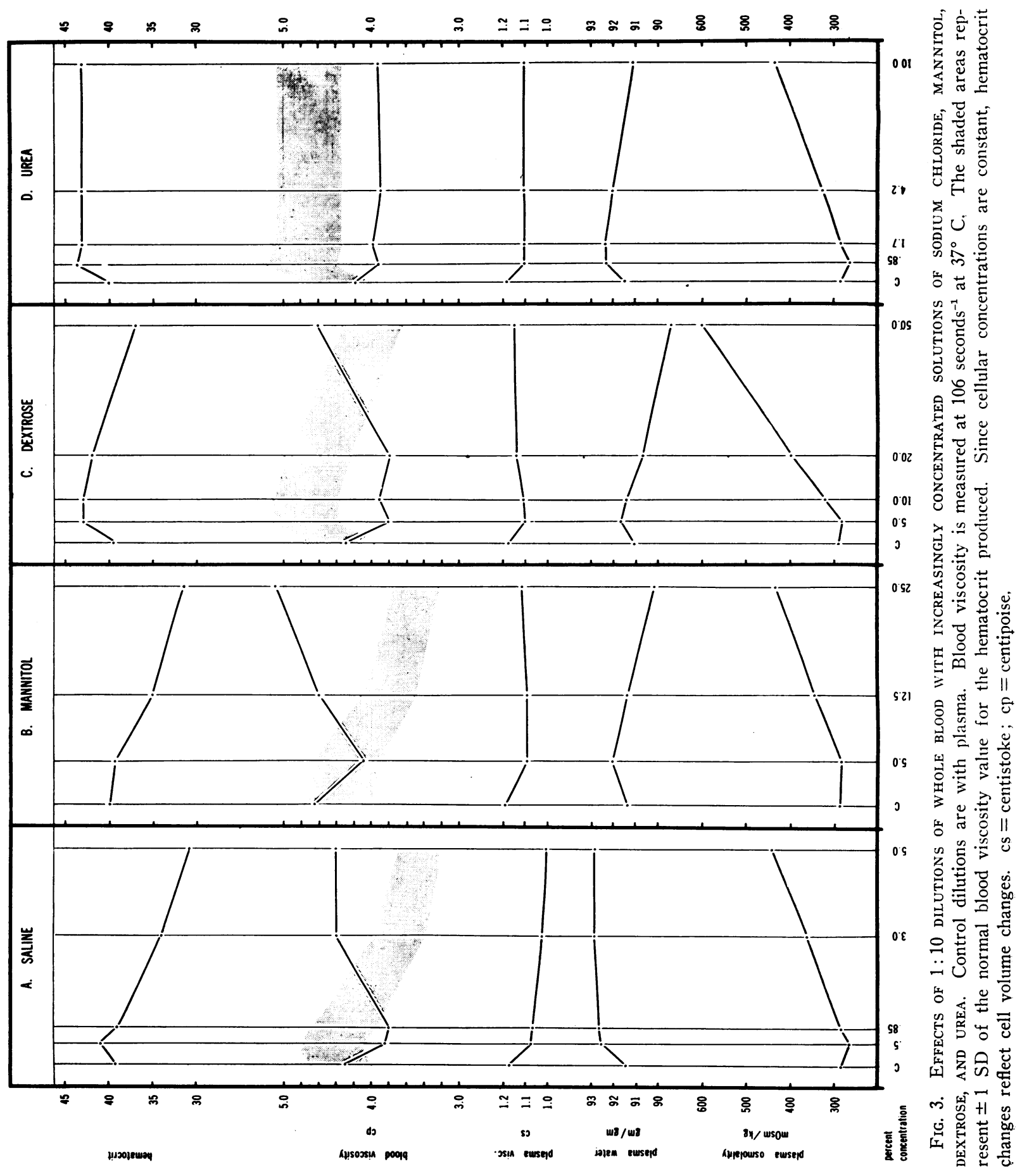


ured cryoscopically with a Fiske osmometer. Hematocrits were determined by the Wintrobe technique.

\section{Results}

Isotonic and hypotonic dilution (Figure 1). When increasing volumes of either plasma or isotonic saline are added to whole blood, a predictable linear drop in hematocrit occurs. With saline dilution, however, plasma undergoes an increase in water concentration, a decrease in protein concentration, and a fall in viscosity. As a result, the viscosity of this blood remains lower than that of whole blood equally diluted with plasma.

Although the same plasma changes take place when equivalent dilutions are made with deionized water, the fall in hematocrit is delayed due to osmotic swelling of the red cells. Despite this increase in red cell size, viscosity decreases to the same extent as it does with equal isotonic dilution. Further examples of decreased viscosity in the face of hypotonic dilutions and osmotically distended cells are provided by hypotonic dilutions of solutes tested in the subsequent series of experiments (see Figure 3 ).

Hypertonic dilution. In hypertonic concentrations, each of the solutes used in this study differs in its effect on red cell size. This is best demonstrated by noting the change in hematocrit that results from equal dilution of identical blood samples with increasingly concentrated solutions. These relationships are presented in Figure 2. As osmolality increases, the hematocrit response will depend on the permeability of the red cell membrane to the particle in question. If it is freely diffusible, as in the case of urea, no osmotic gradients will prevail, and cell size will not diminish ; if it cannot enter the cells, as exemplified by mannitol, water will be extracted, cells will crenate, and hematocrit will fall.

Saline (Figure 3A). When increasingly concentrated samples of saline are used to make $1: 10$ dilutions with whole blood, hematocrit drops rapidly. As has been previously shown, dilutions in the hypotonic and isotonic range make the blood less viscid. Despite the continuing fall in hematocrit, however, blood viscosity begins to rise as the plasma is made more hypertonic. The viscous effect of aqueous plasma dilution is overcome as plasma osmolality exceeds $350 \mathrm{mOsm}$ per $\mathrm{kg}$. Beyond this point the viscosity of these mixtures remains well above the levels that would be expected in undiluted blood at similar hematocrits. Sodium chloride appears to differ from other compounds tested in that it fails to produce a further rise in viscosity at extreme degrees of hypertonicity.

Mannitol (Figure 3B). As crenation is produced by increasingly hypertonic solutions of mannitol, hematocrit falls, and, as in the studies with sodium chloride, viscosity rises. With mannitol the viscosity rise continues in the extremely hypertonic samples. No change in the upward slope of the viscosity curve was seen with plasma osmolalities as high as $600 \mathrm{mOsm}$ per $\mathrm{kg}$. The larger size of the mannitol molecule creates a slight increase in plasma viscosity and a decrease (by displacement) of plasma water. Neither of these changes seems of sufficient magnitude to affect whole blood viscosity.

Dextrose (Figure 3C). Since the red cell membrane is, to a degree, permeable to dextrose, crenation will not occur until plasma osmolality has been elevated to moderately hypertonic levels by the addition of increasingly concentrated solutions. While the red cells are swollen with added dextrose and water, blood viscosity remains depressed by plasma dilution. After exposure to plasma above $400 \mathrm{mOsm}$ per $\mathrm{kg}$, crenation occurs and blood viscosity increases. As in the case of mannitol, the rise is linear and remains so in extremely hypertonic samples (953 mOsm per $\mathrm{kg}$ ). Changes in plasma water and viscosity again do not seem sufficient to explain the degree of blood viscosity change.

Urea (Figure 3D). Although urea is an osmotically active particle, it is freely diffusible across the red cell membrane and therefore will not produce crenation when present in hypertonic concentrations in the plasma. The rise in hematocrit observed in the urea dilutions is proportional not to its concentration (since no osmotic gradients are formed) but to the degree to which its aqueous solvent dilutes the plasma. The hematocrit remains at a constant level above the control as plasma osmolality increases to at least $600 \mathrm{mOsm}$ per $\mathrm{kg}$ (reached in the $1: 5$ dilution). With no crenation occurring despite hypertonicity, 


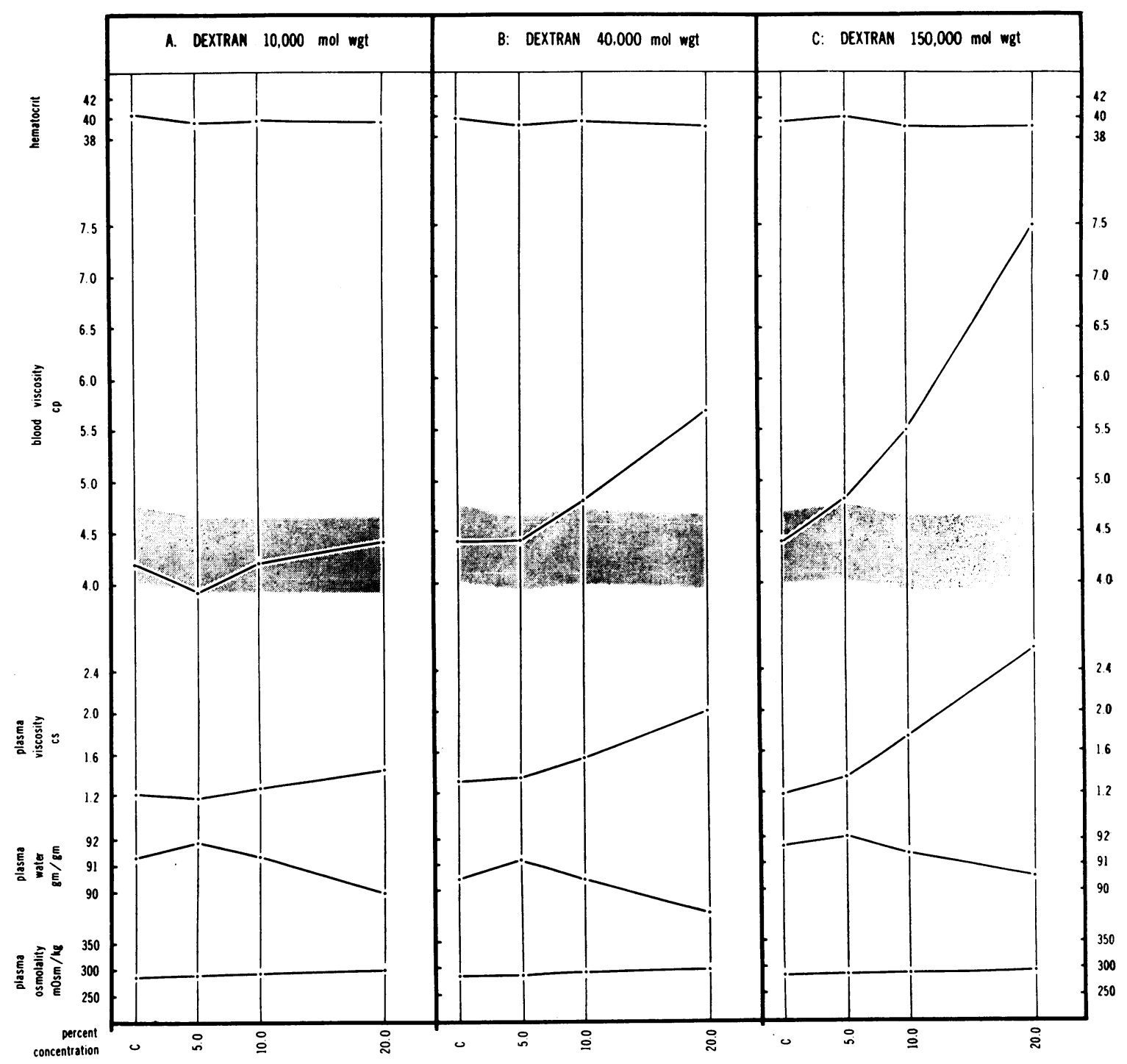

Fig. 4. EFFECTS of 1:10 ISOTONIC DILUTIONS OF WHOLE BLOOD WITH INCREASINGLy VISCID DEXTRAN SOLUTIONS. Data are presented as in Figure 3.

the viscosity of the blood remains at its diluted level well below the control values. Again, changes in plasma viscosity are unremarkable.

Hypertonicity and hematocrit. When red cell concentrations in normal blood exceed $50 \%$, viscosity values rise sharply. The viscous response to hypertonic dilution might be expected to be considerably greater as hematocrits increase. To explore this point, we compared the changes in blood viscosity caused by diluting $(1: 10)$ samples of blood at different hematocrits with $5 \%$ saline. Initial hematocrits were adjusted so that after equal isotonic dilution (control) the per cent vol- ume of red cells would approach either 40 or 50 . The results of this study demonstrate (Table III) that the increase in viscosity after hypertonic dilution is indeed greater at higher hematocrits. Furthermore, this change takes place despite a drastic reduction in hematocrit secondary to crenation.

Dilution with dextrans (Figure 4). In previous experiments whole blood viscosity has been changed by osmotic effects. By using dextrans of various molecular weights, solutions were made up with physiologic saline that were essentially isotonic (hematocrits remained constant) yet 


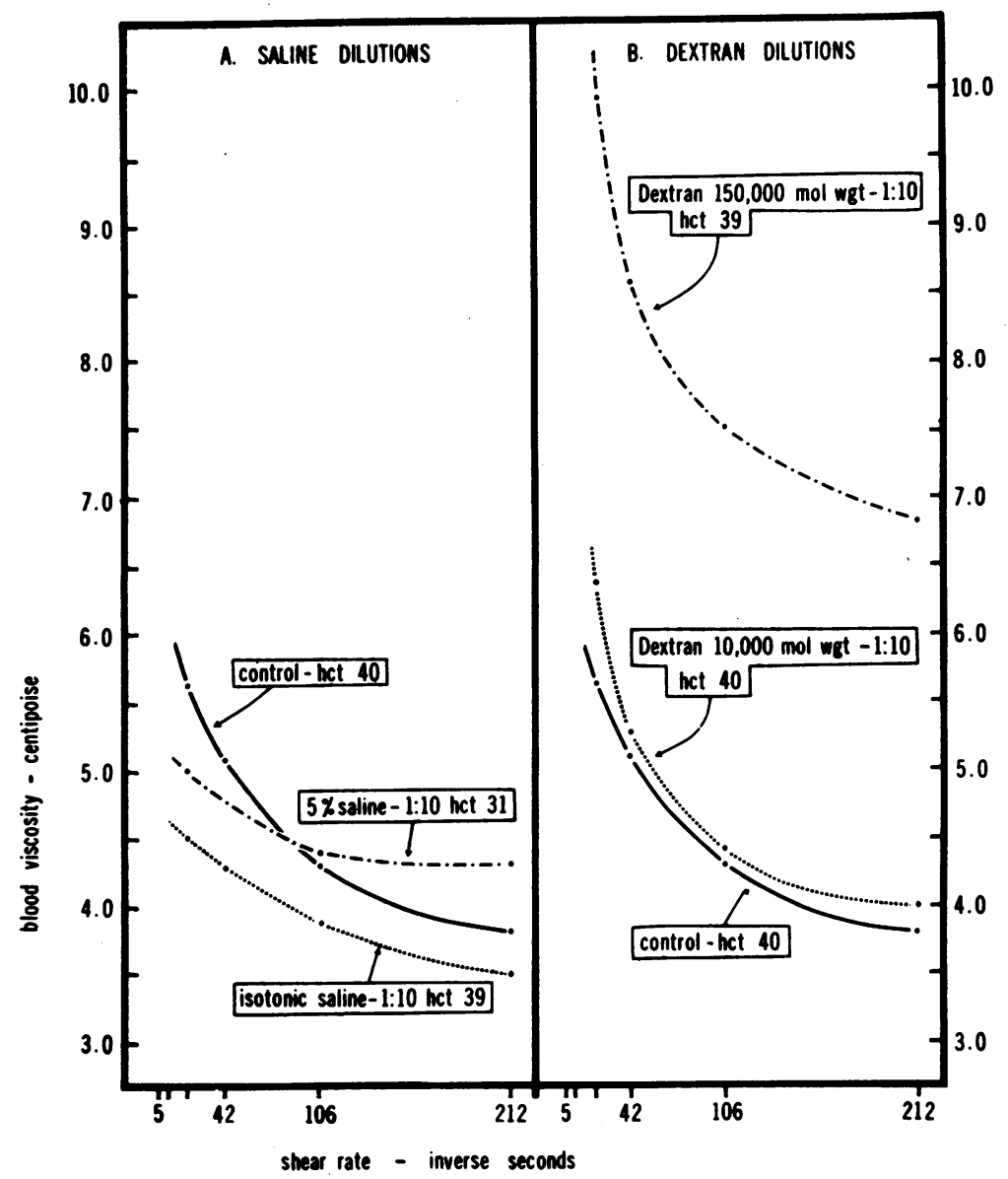

Fig. 5. Shear-Rate dependence of diluted blood. A) Effects of isoTONIC AND HYPERTONIC SOLUTIONS. B) EFFECTS OF ISOTONIC DILUTION WITH LOW AND HIGH MOlecular Weight Dextrans. Control samples in each study are equal dilutions with plasma. het $=$ hematocrit.

varied greatly in viscosity. By diluting blood with these solutions we hoped to examine the relationship between plasma viscosity and whole blood viscosity.

The rise in plasma viscosity, even in the low molecular weight dextran dilutions, was marked in comparison to the previous studies. Blood viscosity showed the effects of aqueous dilution only in the $5 \%$ concentrations of $10,000 \mathrm{~mol} \mathrm{wt}$ dextran. In all other cases it demonstrated a marked rise, the slope of which increased with the molecular weight of the dextran employed. Since the dextrans are thought to have significant though varying effects on the attractive forces between red cells $(18,19)$, it should not be concluded that their effect on whole blood viscosity is entirely a reflection of the changes in plasma viscosity.

Shear-rate dependence (Figure 5). The prop-

TABLE III

Effect of hematocrit on response of blood viscosity to hypertonicity

\begin{tabular}{|c|c|c|c|c|}
\hline Diluent & $\underset{\text { crit }}{\text { Initial }}$ & $\begin{array}{c}\text { Hemato- } \\
\text { crit } \\
\text { after } \\
\text { dilution }\end{array}$ & $\begin{array}{c}\text { Blood } \\
\text { viscosity } \\
106 \\
\text { seconds }^{-1}\end{array}$ & $\underset{\text { Viscosity }}{\Delta}$ \\
\hline & & & centipoise & centipoise \\
\hline Plasma & $\begin{array}{l}44.4 \\
55.5\end{array}$ & $\begin{array}{l}40 \\
50\end{array}$ & $\begin{array}{l}4.4 \\
4.7\end{array}$ & 1.3 \\
\hline \multirow{2}{*}{$\begin{array}{c}\text { Isotonic } \\
\text { saline } \\
1: 10\end{array}$} & 44.4 & 39 & 3.8 & \multirow[t]{2}{*}{1.0} \\
\hline & 55.5 & 49 & 4.8 & \\
\hline \multirow{2}{*}{$\begin{array}{l}\text { Hypertonic } \\
\text { saline } \\
1: 10\end{array}$} & 44.4 & 31 & 3.4 & \multirow[t]{2}{*}{3.5} \\
\hline & 55.5 & 40 & 6.9 & \\
\hline
\end{tabular}


erty of whole blood to become rapidly more viscid at low shear rates is related primarily to the concentration of red cells (20), secondarily to the relative concentrations of the plasma proteins $(21,22)$. With the addition of isotonic fluid, therefore, the viscosity-shear-rate curves tend to be lower and more gradual as both cells and plasma are diluted. We have repeatedly observed the same response in blood with hypertonic plasma whether osmolality has been increased by dilution with concentrated solutes or by the addition of dry sodium chloride. It may be pertinent that both methods increase the absolute amount of water in the plasma from either exogenous or endogenous (extracted cell water) sources and that plasma viscosity is diminished. Dilution with $10,000 \mathrm{~mol}$ wt dextran did little to the sheardependent character of whole blood, but dilution with high molecular weight $(150,000)$ dextran produced greatly elevated whole blood viscosity values that also demonstrated an increase in shear-rate dependence at low shear rates.

\section{Discussion}

The significant degree to which whole blood viscosity is affected by plasma proteins has been studied by Wells, Merrill, and Gabelnick (21), who point out that dilution of proteins reduces whole blood viscosity both by decreasing plasma viscosity and by diminishing protein-induced red cell aggregation [the "viscosity plus factor" of Gelin (23) and the "second heterophase effect" of Merrill and co-workers (24)]. Our data demonstrate that the viscosity-reducing effects of isotonic and hypotonic dilution are similar, despite the osmotic increase in red cell size in hypotonic plasma. These results should be compared with those of Gregersen and associates (25), who demonstrated an increase in viscosity with increasing cell size $\left(20 \mu^{3}\right.$ to $\left.114 \mu^{3}\right)$ using blood samples of equal hematocrit from several mammalian species.

Although the decrease in blood viscosity after hypotonic dilution is satisfactorily explained on the basis of plasma protein dilution, one might speculate that the osmotically distended spheroidal cells present less surface area for intercellular contact than biconcave discs and thus offer less resistance to flow. If this were the case, however, the viscosity levels after equivalent isotonic and hypotonic dilutions would not have agreed so closely. It should also be noted, in view of recent work $(26,27)$ suggesting a correlation among intracellular viscosity, mean cellular hemoglobin concentrations, and whole blood viscosity, that despite the decrease in mean corpuscular hemoglobin concentration produced in our hypotonic samples, the drop in blood viscosity was not significantly greater than in isotonic samples. Our findings do not agree with those of Wells, Cox, and Shahriari (28), who noted an increased blood viscosity with hypotonic dilution.

One might expect the addition of diluents of high osmolality to decrease blood viscosity because of the combined protein-diluting effects of both exogenous water and extracted cell water. Blood viscosity rises, however, and a comparison of the response to permeant (urea) and nonpermeant (saline, mannitol) solutes strongly suggests that this rise is due to crenation. We have been unable to find previous reports of this effect, although Lilien, Jones, and Mueller (29) observed crenation, a drop in hematocrit, and decreased whole blood viscosity after the injection of hypertonic mannitol into human and canine subjects. Water drawn in from the extravascular spaces probably provided sufficient dilution to explain the diminished viscosity they noted.

Because of their irregular surfaces, it seems obvious that crenated cells would offer more resistance to movement than normal cells if both were present in the same volume concentration. Both Erslev and Atwater (26) and Dintenfass (27) have presented evidence that the viscosity of blood is affected by the elastic deformability of the red cell, which is dependent in turn on the viscosity of the cell contents. Hypertonic nonpermeant solutes, by removing cell water, would increase intracellular viscosity.

In a separate study to explore this point we measured the viscosity and water contents of intracellular material obtained by freeze-thaw lysis and centrifugation of red cells from blood samples exposed to various concentrations of solutes previously mentioned. As would be expected, hypertonicity decreased cell water and increased the viscosity of the cellular contents.

Although the information from this study sup- 
ports the concept that at equal hematocrits crenated cells should diminish blood flow more than normal cells, we are unable to explain the conspicuous influence of crenation on blood viscosity in the face of marked plasma and red cell dilution.

Our use of dextran was intended not so much to imitate a clinical situation as to evaluate the effect of plasma viscosity changes on whole blood viscosity. Previous work (13) has shown that blood viscosity increases with an increase in the molecular weight of the added dextran. Recent measurements with variable shear-rate viscometers have provided evidence that this increase is more than would be expected from changes in plasma viscosity alone and that the rise in blood viscosity is more pronounced at low shear rates $(23,30)$.

Classic studies by Swedish workers (18) revealed that high molecular weight dextran causes red cell aggregation, whereas low molecular weight dextran reverses or prevents it. Microelectrophoretic evidence $(19,31)$ suggests that this phenomenon involves changes in the charge of the red cell membrane. What is of primary interest to us is the degree to which plasma viscosity must be raised in a relatively isotonic medium to achieve blood viscosity elevations equal to those produced in hypertonic media. This information nullifies the significance of the slight shifts in plasma viscosity seen in our electrolyte and crystalloid dilutions and provides a frame of reference for studies involving highly viscous radiographic dyes to be mentioned. A satisfactory explanation for the effects of the dextrans and other macromolecular diluents on blood rheology will require considerable further investigation.

Whereas the addition of large molecules to blood tends to make its viscosity more shear-rate dependent, the addition of hypertonic solutes, which dilute plasma proteins, appears to flatten viscosity-shear-rate curves. Shear-rate dependence is more responsive to changes in plasma composition than to changes in cell size or shape. Our viscometer lacked sufficient sensitivity at shear rates low enough to explore this point adequately, but the implication would be that the increased viscosity of hypertonic blood would not rise to substantially higher values as shear rate decreased. In the postcapillary venules, where shear rate may be low, this blood should not impede flow to the same extent as normal blood with equivalent viscosity at high shear rates.

Clinical implications. The establishment of total body perfusion using an oxygenator primed either with $5 \%$ dextrose in water or physiologic electrolyte solution provides the most dramatic clinical example of isotonic or hypotonic blood dilution. In comparison with perfusions using whole blood only, venous return is increased, higher flow rates may be achieved, and blood pressure is more uniform (32). Cooley, Beall, and Grondin (10) report less aggregation and blood sequestration in the microcirculation, improved renal function, and fewer cerebral, pulmonary, and renal complications resulting from hemodilution perfusion. Although these signs of improved peripheral blood flow may all stem directly or indirectly from changes in the physical properties of blood, this point cannot be proved convincingly until the relationship of blood viscosity to flow through the microcirculation is more clearly established.

Although high molecular weight dextrans have not been found safe for clinical use, dextrans with molecular weights of 40,000 or less have received much attention because of their ability to prevent intravascular aggregation of red cells and because of the beneficial responses said to result from their clinical use. Since isotonic saline or 5\% dextrose in water is used as a vehicle for dextran infusion, we feel that dilution alone may figure importantly in these effects. This point certainly requires further clarification.

Hypertonic medications such as $50 \%$ dextrose in water or concentrated sodium bicarbonate are usually administered in such small volumes and at such slow rates as to preclude hemodynamically important systemic effects. It has been observed that the extended infusion of hypertonic solutions of nonpermeant particles such as mannitol produce decreased blood viscosity and increased flow in vivo, at least as measured through the kidney $(29,33,34)$. Dilutions with water from the extravascular spaces as well as from the red cells probably explain these findings. More rapid injections of hypertonic solutions, however, have been shown repeatedly to produce pulmonary hypertension and peripheral hypotension, the response being proportional to the concentration and 


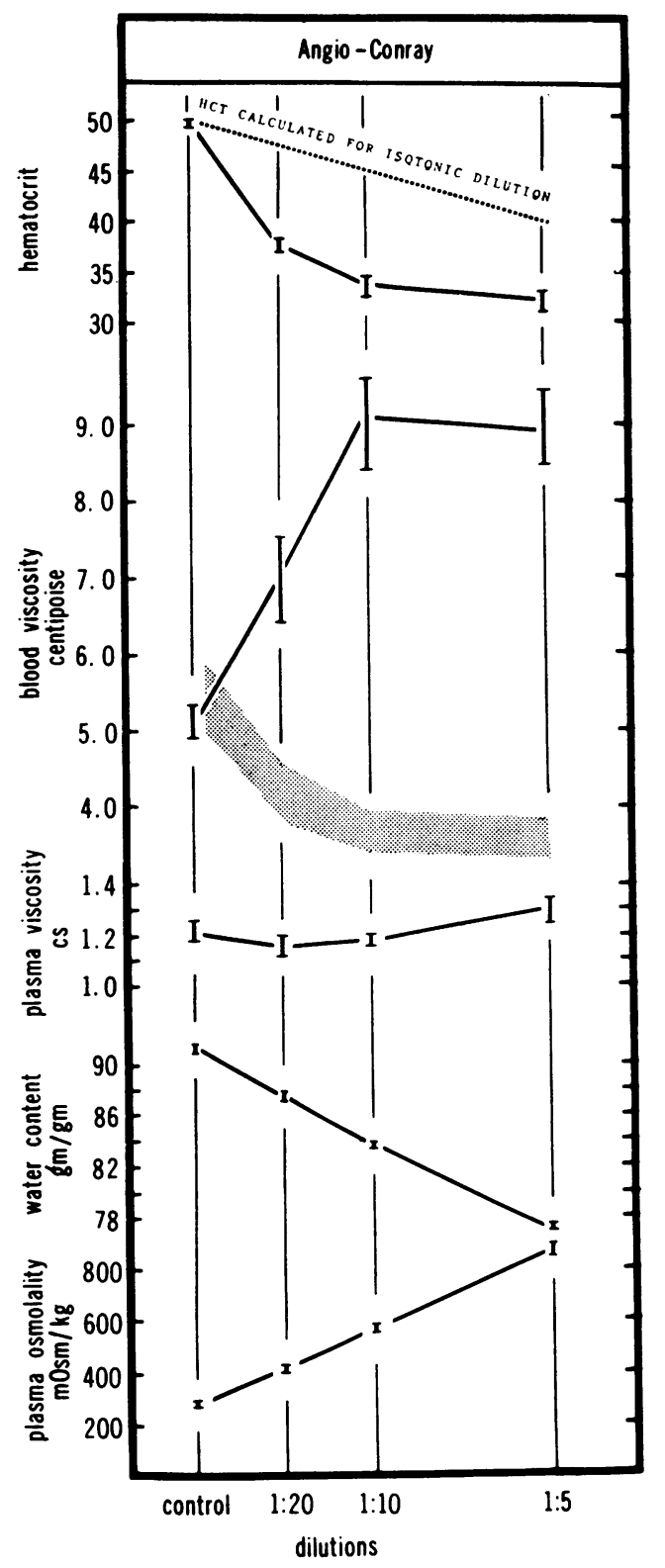

Fig. 6. EFFects of diluting whole blood With iNCREASing amounts of Angio-Conray. Data are presented as in Figure 1, except that the control samples in this instance are normal blood at hematocrit 50 . The range of values from all studies is indicated by vertical lines at each dilution.

speed of injection of the test fluid. Only urea, which is freely diffusible across the red cell membrane, fails to produce these reactions. Although there is general agreement that neurovascular reflexes do not play a part in this phenomenon, opinions are not uniform regarding the site of action. Muirhead, Lackey, Bunde, and Hill (1) suggest that the response takes place primarily within the myocardium, whereas Eliakim, Rosenberg, and Braun (2) feel that spasm of the pulmonary vein-left atrial junction is responsible. The investigations of Semler, Shepherd, and Swan (3) and of Read, Johnson, Vick, and Meyer (4) indicate that the obstruction to flow is in the pulmonary vascular bed. Read and associates feel that the pulmonary hypertensive effect depends upon changes in blood viscosity rather than upon vascular changes, and support this belief by microscopic evidence of red cell clumping and stagnated flow in the microcirculation after injection of hypertonic saline. We feel that the present demonstration of increasing viscosity with crenation adds strength to this thesis and extends understanding of the mechanisms involved.

Cardioangiography provides the clinical counterpart to the investigations just discussed. Radiopaque agents are highly viscous and extremely hypertonic, yet good visualization requires that as large a dose as possible be injected as rapidly as possible. This combination of factors might be expected to contribute to a marked rise in blood viscosity. Hemodynamic responses to angiographic dye injection, which are parallel to those seen after hypertonic injections, have been extensively studied by Read (6), who observed reactions sufficiently severe to cause death from acute cor pulmonale. Recently his findings have been confirmed and extended by Austen, Wilcox, and Bender (35).

In an additional study we attempted to demonstrate the effect of the radiographic dye AngioConray (sodium iothalamate $80 \%$ ) $^{2}$ on blood viscosity by studying increasing dilutions of whole blood with the dye (experiment performed in quadruplicate. See Figure 6). The initial hematocrit of the control samples was 50 . The addition of $1 \mathrm{ml}$ of Angio-Conray to $9 \mathrm{ml}$ of this blood produced marked crenation resulting in a fall of hematocrit far below that which would be expected with equal isotonic dilution. Despite dilution, however, blood viscosity rose to levels twice as high as would be expected at the hematocrits produced. Further dilution with this compound, which, by itself, has the same viscosity at

2 Mallinkrodt Chemical Works, St. Louis, Mo. 
106 seconds $^{-1}$ as whole blood of hematocrit 65 , produced no further drop in hematocrit or rise in viscosity.

To have an effect in the pulmonary circulation after injection into the right ventricle, the changes we have noted must occur within seconds. To establish this point, a hemocytometer chamber was partially filled with a suspension of red cells in plasma. Rapid serial photographs were then taken as a drop of Angio-Conray was introduced under the coverslip. Exposures were made at 2 -second intervals starting with the addition of the dye. On all occasions crenation had taken place by the time cell movement had slowed sufficiently to observe it, an interval of less than 2 seconds.

This information emphasizes two points regarding the effects of angiographic agents on blood flow in vivo. 1) Viscosity is greatly increased by dilutions that are usually equalled or exceeded clinically, and 2) the viscous effects are magnified by increasing red cell concentration (Figure 4). The implications are that these agents should be used with caution in the presence of polycythemia and especially in the presence of pulmonary vascular disease.

\section{Summary}

In this study viscosity changes have been measured in whole blood after in vitro dilution with varying concentrations of several intravenous agents. In the presence of fixed red cell concentrations, the following responses have been noted:

1) Isotonic dilution depresses both plasma and whole blood viscosity.

2) Hypotonic dilution produces the same effects despite osmotic swelling of the red cells and a consequent rise in hematocrit.

3) Hypertonic dilution causes a marked rise in blood viscosity if crenation is produced; hypertonic concentrations of diffusible solutes (urea) produce neither crenation nor a rise in blood viscosity.

4) The rise in viscosity with crenation is not due to increased plasma viscosity and takes place despite a marked decrease in hematocrit.

5) The dependence of blood viscosity on shear rate is reduced by both hypotonic and hypertonic saline or mannitol dilution but accentuated by di- lution with dextrans of increasing molecular weight.

Physiologic implications of these changes are discussed. The response to angiographic dye dilution is presented to emphasize their clinical significance.

\section{Acknowledgments}

The authors wish to express their appreciation to Mrs. Nancy Barker, Miss Joan Gooch, and Mr. James McKenzie for technical, secretarial, and photographic assistance. We are indebted to Dr. Manu Chatterjee for his critical review of the manuscript.

\section{References}

1. Muirhead, E. E., R. W. Lackey, C. A. Bunde, and J. M. Hill. Transient hypotension following rapid intravenous injections of hypertonic solutions. Amer. J. Physiol. 1947, 151, 516.

2. Eliakim, M., S. Z. Rosenberg, and K. Braun. Effect of hypertonic saline on the pulmonary and systemic pressures. Circulat. Res. 1958, 6, 357.

3. Semler, H. J., J. T. Shepherd, and H. J. C. Swan. Effect of hypertonic saline solution on the pulmonary circulation (abstract). Physiologist 1958, 1, 71.

4. Read, R. C., J. A. Johnson, J. A. Vick, and M. W. Meyer. Vascular effects of hypertonic solutions. Circulat. Res. 1960, 8, 538.

5. Cotrim, E. S. Cardiac, blood pressure, and respiratory effects of some contrast media: the dangers of overdosage. Acta radiol. (Stockh.) 1954, 116, 58.

6. Read, R. C. Cause of death in cardioangiography. J. thorac. cardiovasc. Surg. 1959, 38, 685.

7. Bernstein, E. F., and R. L. Evans. Low-molecularweight dextran. J. Amer. med. Ass. 1960, 174, 1417.

8. Long, D. M., Jr., L. Sanchez, R. L. Varco, and C. W. Lillehei. The use of low molecular weight dextran and serum albumin as plasma expanders in extracorporeal circulation. Surgery 1961, 50, 12.

9. Zuhdi, N., B. McCollough, J. Carey, and C. Krieger. Hypothermic perfusion for open-heart surgical procedures. J. int. Coll. Surg. 1961, 35, 319.

10. Cooley, D. A., A. C. Beall, Jr., and P. Grondin. Open-heart operations with disposable oxygenators, 5 per cent dextrose prime, and normothermia. Surgery 1962, 52, 713.

11. Roe, B. B., S. A. Hepps, and E. E. Swenson. Hemodilution with and without low-mole dextran : laboratory studies and clinical experience (abstract). Circulation 1963, 28, 792.

12. DeWall, R. A., R. C. Lillehei, and R. D. Sellers. Hemodilution perfusions for open-heart surgery. New Engl. J. Med. 1962, 266, 1078. 
13. Reemtsma, K., and O. Creech, Jr. Viscosity studies of blood, plasma, and plasma substitutes. J. thorac. cardiovasc. Surg. 1962, 44, 674.

14. DeWall, R. A., and C. W. Lillehei. Simplified total body perfusion. Reduced flows, moderate hypothermia, and hemodilution. J. Amer. med. Ass. 1962, 179, 430.

15. Rand, P. W., E. Lacombe, H. E. Hunt, and W. H. Austin. Viscosity of normal human blood under normothermic and hypothermic conditions. J. appl. Physiol. 1964, 19, 117.

16. Merrill, E. W., G. C. Cokelet, A. Britten, and R. E. Wells, Jr. Non-Newtonian rheology of human blood-effect of fibrinogen deducted by "subtraction." Circulat. Res. 1963, 13, 48.

17. Hardy, R. C. NBS Viscometer Calibrating Liquids and Capillary Tube Viscometers. National Bureau of Standards Monograph 55, 1962.

18. Thorsen, G., and H. Hint. Aggregation, sedimentation and intravascular sludging of erythrocytes. Acta chir. scand. (suppl.) 1950, 154.

19. Bernstein, E. F., F. G. Emmings, G. C. Mackey, A. Castaneda, and R. L. Varco. Effect of low molecular weight dextran on red blood cell charge during extracorporeal circulation. Trans. Amer. Soc. Artif. Organs 1962, 8, 23.

20. Wells, R. E., Jr., and E. W. Merrill. Influence of flow properties of blood upon viscosity-hematocrit relationships. J. clin. Invest. 1962, 41, 1591.

21. Wells, R. E., Jr., E. W. Merrill, and H. Gabelnick. Shear-rate dependence of viscosity of blood: interaction of red cells and plasma proteins. Trans. Soc. Rheology 1962, 6, 19.

22. Wells, R. E., Jr., R. D. Perera, T. Gawronski, and A. A. Shahriari. Effects of plasma proteins upon the rheological character of blood in the microcirculation (abstract). J. clin. Invest. 1963, 42, 991.

23. Gelin, L. E. Rheological disturbances following tissue injury. Abstracts of the Fourth International Congress on Rheology. Brown University, 1963, 244.
24. Merrill, E. W., E. R. Gilliland, G. Cokelet, H. Shin, A. Britten, and R. E. Wells, Jr. Rheology of blood and flow in the microcirculation. J. appl. Physiol. 1963, 18, 255.

25. Gregersen, M. I., B. Peric, S. Chien, D. Sinclair, C. Chang, and $\mathrm{H}$. Taylor. The influence of erythrocyte size on the viscosity of blood at low shear rates. Abstracts of the Fourth International Congress of Rheology. Brown University, 1963, 279.

26. Erslev, A. J., and J. Atwater. Effect of mean corpuscular hemoglobin concentration on viscosity. $\mathrm{J}$. Lab. clin. Med. 1963, 62, 401.

27. Dintenfass, L. Considerations of the internal viscosity of red cells and its effect on the viscosity of whole blood. Angiology 1962, 13, 333.

28. Wells, R. E., Jr., P. J. Cox, Jr., and A. A. Shahriari. Effects of $\mathrm{pH}$ and osmolality upon blood viscosity. Clin. Res. 1963, 11, 176.

29. Lilien, O. M., S. G. Jones, and C. B. Mueller. The mechanism of mannitol diuresis. Surg. Gynec. Obstet. 1963, 117, 221.

30. Gregersen, M. I., S. Usami, B. Peric, C. Chang, D. Sinclair, and S. Chien. Blood viscosity at low shear rates. Effects of low and high molecular dextrans. Biorheology 1963, 1, 247.

31. Bernstein, E. F., F. G. Emmings, R. L. Evans, A. Castaneda, and R. L. Varco. Effect of low molecular weight dextrans on red blood cell charge during clinical extracorporeal circulation. Circulation 1963, 27, 816.

32. Zuhdi, N., J. Carey, J. Cutter, L. Rader, and A. Greer. Intentional hemodilution. Arch. Surg. 1963, 87, 554.

33. Koch, A., A. M. Scher, and J. P. McDonald. Effects of tonicity of perfusion fluid and urine flow on renal vascular resistance. Fed. Proc. 1963, 22, 172.

34. Gagnon, J. A., G. P. Murphy, and P. E. Teschan. Renal hemodynamic effects of hypertonic mannitol in the dog. Fed. Proc. 1963, 22, 173.

35. Austen, W. G., B. R. Wilcox, and H. W. Bender. Experimental studies of the cardiovascular responses secondary to the injection of angiographic agents. J. thorac. cardiovasc. Surg. 1964, 47, 356. 